\title{
Variation of magnetic energy in oriented ultra-thin ferromagnetic films
}

\author{
P. Samarasekara \\ Department of Physics, University of Ruhuna, Matara, Sri Lanka, pubudus@phy.ruh.ac.lk
}

\begin{abstract}
The angle dependence of magnetic energy of ultra-thin films with $\mathrm{N}=1$ to 5 layers has been investigated. The effect of stress and the demagnetization factor on the classical Heisenberg Hamiltonian has been taken into account in addition to other magnetic factors. Films of $\mathrm{sc}(001)$ and bcc(001) ferromagnetic indicate preferred in plane and perpendicular orientations for $\mathrm{N} \leq 3$ and $\mathrm{N} \geq 4$, respectively. Easy and hard directions of all $\mathrm{sc}(001)$, bcc(001) and fcc(001) films with five layers are $\theta=7.2^{\circ}$ and $\theta=114^{\circ}$ respectively. According to our simulation, ultra-thin films with one or two layers can be easily oriented in $\theta=279^{\circ}$ direction.

Key words: Spin, magnetic moment, magnetic anisotropy, ferromagnetic materials, thin films, Heisenberg Hamiltonian
\end{abstract}

\section{Introduction.}

Although the experimental evidence of oriented and non-oriented ferromagnetic and ferrimagnetic films can be found in many early reports, the theoretical aspects of oriented ferrimagnetic films were not revealed in a considerable manner yet. The sputter synthesis of aligned strontium hexaferrites, $\mathrm{SrO} .6\left(\mathrm{Fe}_{2} \mathrm{O}_{3}\right)$, films can be given as an example of the deposition of oriented ferromagnetic film (Hegde et al. 1994a). The sputter synthesis of $\mathrm{TbCu}_{7}$ type $\mathrm{Sm}(\mathrm{CoFeCuZr})$ films with controlled easy axis orientation (Hegde et al. 1994b) and nitriding studies of aligned high anisotropy $\mathrm{ThMn}_{12}$-type $\mathrm{NdFe}_{11} \mathrm{Co}_{1-y} \mathrm{Mo}_{y} \mathrm{~N}$ film samples can be found in some experimental reports of oriented films (Navarathna et al. 1994). Easy axis oriented Nickel ferrite (Samarasekara et al. 1996) and Lithium mixed ferrite (Samarasekara and Cadieu 2001) thin films have been fabricated on single crystal substrates by means of pulse laser deposition technique.

The magnetic properties of ferromagnetic films have been investigated using Bloch-spin wave theory (Martin and Robert 1951). Monte Carlo simulation of hysteresis loops of $\mathrm{FeF}_{2}(110) / \mathrm{Fe}$ bilayers has been performed to study the exchange interaction between antiferromagnetic and ferromagnetic sublattices (Lederman et al. 2004). The Energy of oriented ferromagnetic thick films has been calculated using the Heisenberg Hamiltonian in one of our early report. The energy of oriented ultra-thin ferromagnetic films will be described in this report. The effect of spin exchange interaction, magnetic dipole interaction, anisotropies up to eighth order, demagnetization factor and stress induced anisotropy on Heisenberg Hamiltonian was studied. 


\section{Model and discussion}

The classical Heisenberg Hamiltonian for ferromagnetic thin films can be given as following.

$$
\begin{aligned}
H & =-\frac{J}{2} \sum_{m, n} \vec{S}_{m} \cdot \vec{S}_{n}+\frac{\omega}{2} \sum_{m \neq n}\left(\frac{\vec{S}_{m} \cdot \vec{S}_{n}}{r_{m n}^{3}}-\frac{3\left(\vec{S}_{m} \cdot \vec{r}_{m n}\right)\left(\vec{r}_{m n} \cdot \vec{S}_{n}\right)}{r_{m n}^{5}}\right) \\
& -\sum_{m} D_{\lambda_{m}}^{(2)}\left(S_{m}^{z}\right)^{2}-\sum_{m} x D_{\lambda_{m}}^{(4)}\left(S_{m}^{z}\right)^{4} \\
& -\sum_{m}^{m} D_{\lambda_{m}}^{(6)}\left(S_{m}^{z}\right)^{6}-\sum_{m}^{m} D_{\lambda_{m}}^{(8)}\left(S_{m}^{z}\right)^{8} \\
& -\sum_{m, n}^{m}\left[\vec{H}-\left(N_{d} \vec{S}_{n} / \mu_{0}\right)\right] \cdot \vec{S}_{m}-\sum_{m} K_{s} \sin 2 \theta_{m}
\end{aligned}
$$

Above equation for oriented ferromagnetic thin films can be deduced to following form (Usadel and Hucht 2002).

$$
\begin{aligned}
E(\theta) & =-\frac{1}{2} \sum_{m, n=1}^{N}\left(J Z_{|m-n|}-\frac{\omega}{4} \Phi_{|m-n|}\right)+\frac{3 \omega}{8} \cos 2 \theta \sum_{m, n=1}^{N} \Phi_{|m-n|} \\
& -\cos ^{2} \theta \sum_{m=1}^{N} D_{m}^{(2)}-\cos ^{4} \theta \sum_{m=1}^{N} D_{m}^{(4)} \\
& -\cos ^{6} \theta \sum_{m=1}^{N} D_{m}^{(6)}-\cos ^{8} \theta \sum_{m=1}^{N} D_{m}^{(8)} \\
& -N\left(H_{\text {in }} \sin \theta+H_{\text {out }} \cos \theta-\frac{N_{d}}{\mu_{0}}+K_{5} \sin 2 \theta\right)
\end{aligned}
$$

Here $J, D_{m}^{(2)}, D_{m}^{(4)}, D_{m}^{(6)}, D_{m}^{(8)}, N, H_{\text {in }}, H_{\text {out }}, N_{d}, K_{5}, Z_{|m-n|}, \Phi_{|m-n|}, \omega$ and $\theta$ are spin exchange interaction, second, fourth, sixth and eight order anisotropy coefficients, number of layers of the film, in plane applied magnetic field, out of plane applied magnetic field, demagnetization factor, stress induced anisotropy coefficient, number of nearest neighbors between layers, constants related to partial summation of dipole interaction, strength of long range dipole interaction and the angle between the spin and the film normal, respectively. For microscopic anisotropy, sixth and eighth order anisotropies are also induced. For an ultra thin film with few layers, it is reasonable to assume that $D_{m}^{(2)}, D_{m}^{(4)}, D_{m}^{(6)}, D_{m}^{(8)}$ are constants.

Because $Z_{\delta>1}=0, \sum_{m, n=1}^{N} Z_{|m-n|}=N Z_{0}+2(N-1) Z_{1}$.

After assuming that $\Phi_{\delta>1}=0, \sum_{m, n=1}^{N} \Phi_{|m-n|}=N \Phi_{0}+2(N-1) \Phi_{1}$.

Then above equation can be reduced into following form,

$$
\begin{aligned}
E(\theta) & =-\frac{J}{2}\left[N Z_{0}+2(N-1) Z_{1}\right]+\frac{\omega}{8}\left[N \Phi_{0}+2(N-1) \Phi_{1}\right](1+3 \cos 2 \theta) \\
& -N\left[D_{m}^{(2)} \cos ^{2} \theta+D_{m}^{(4)} \cos ^{4} \theta+D_{m}^{(6)} \cos ^{6} \theta+D_{m}^{(8)} \cos ^{8} \theta\right. \\
& \left.+H_{\text {in }} \sin \theta+H_{\text {out }} \cos \theta-\frac{N_{d}}{\mu_{0}}+K_{5} \sin 2 \theta\right]
\end{aligned}
$$




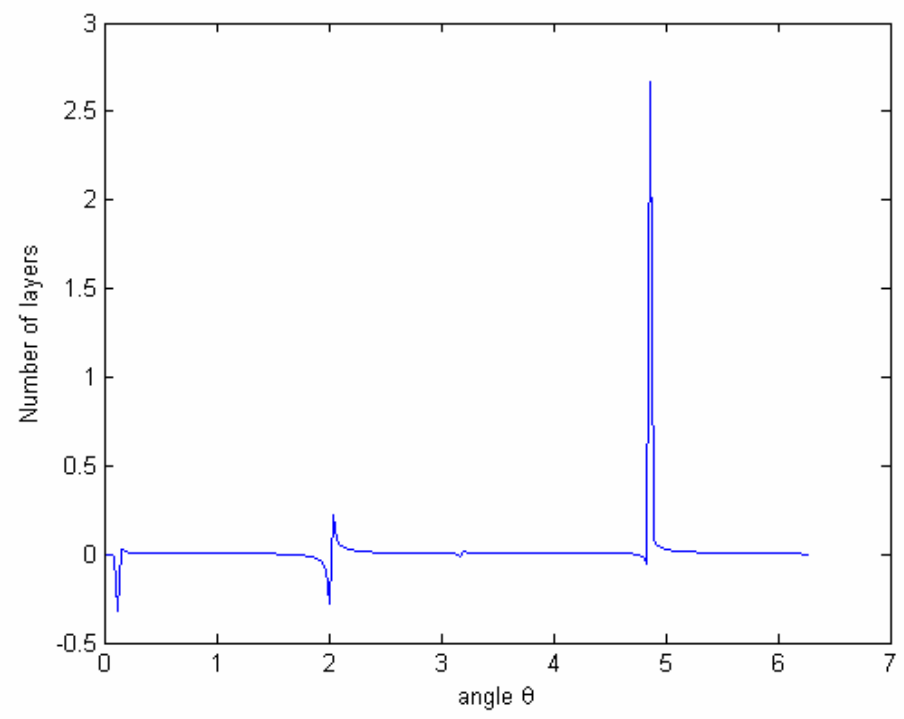

Figure 1 The graph between number of layers and the angle (radians) for minimum energy.

For the minimum energy, $\frac{\partial E}{\partial \theta}=0$, and following equation of $\mathrm{N}$ can be obtained.

$$
N=\frac{1.5 \omega \Phi_{1} \sin 2 \theta}{K}
$$

Here,

$$
\begin{aligned}
K & =0.75 \omega\left(\Phi_{0}+2 \Phi_{1}\right) \sin 2 \theta-2 D_{m}^{(2)} \sin \theta \cos \theta \\
& -4 D_{m}^{(4)} \sin \theta \cos ^{3} \theta-6 D_{m}^{(6)} \sin \theta \cos ^{5} \theta \\
& -8 D_{m}^{(8)} \sin \theta \cos ^{7} \theta+H_{\text {in }} \cos \theta-H_{\text {out }} \sin \theta+2 K_{5} \cos 2 \theta
\end{aligned}
$$

$\frac{K_{5}}{\omega}=10, \frac{D_{m}^{(2)}}{\omega}=30, \frac{D_{m}^{(4)}}{\omega}=20, \frac{D_{m}^{(6)}}{\omega}=10, \frac{D_{m}^{(8)}}{\omega}=5, \frac{H_{\text {in }}}{\omega}=\frac{H_{\text {out }}}{\omega}=10$, and $\frac{N_{d}}{\mu_{0} \omega}=10$ will be used for the simulation in this report.

For sc(001) lattice, $\Phi_{0}=9.0336, \Phi_{1}=-0.3275$ and $N=-\frac{0.4913 \sin 2 \theta}{K}$.

Here $K=-23.716 \sin 2 \theta-20\left(4+3 \cos ^{2} \theta+2 \cos ^{4} \theta\right) \cos ^{3} \theta \sin \theta+10(\cos \theta-\sin \theta)+$ $20 \cos 2 \theta$.

The graph between $\mathrm{N}$ and angle $(\theta)$ in radians is given in Figure 1. The angles indicated in all the graphs are given in radians. According to this graph, films with $\mathrm{N}=1,2$ can be easily oriented in $\theta=279^{\circ}$ direction. Similarly $\mathrm{N}$ corresponding to the minimum energy of fcc(001) and bcc(001) can be found using above equation.

When the summations of $D_{m}^{(2)}, D_{m}^{(4)}, D_{m}^{(6)}, D_{m}^{(8)}$ are considered,

$$
E(\theta)=-\frac{J}{2}\left[N Z_{0}+2(N-1) Z_{1}\right]+\frac{\omega}{8}\left[N \Phi_{0}+2(N-1) \Phi_{1}\right](1+3 \cos 2 \theta)
$$




$$
\begin{aligned}
& -\cos ^{2} \theta \sum_{m=1}^{N} D_{m}^{(2)}-\cos ^{4} \theta \sum_{m=1}^{N} D_{m}^{(4)}-\cos ^{6} \theta \sum_{m=1}^{N} D_{m}^{(6)}-\cos ^{8} \theta \sum_{m=1}^{N} D_{m}^{(8)} \\
& -N\left(H_{\text {in }} \sin \theta+H_{\text {out }} \cos \theta-\frac{N_{d}}{\mu_{0}}+K_{5} \sin 2 \theta\right)
\end{aligned}
$$

When $\theta=0$,

$$
\begin{aligned}
E(0) & =-\frac{J}{2}\left[N Z_{0}+2(N-1) Z_{1}\right]+\frac{\omega}{2}\left[N \Phi_{0}+2(N-1) \Phi_{1}\right] \\
& -\sum_{m=1}^{N} D_{m}^{(2)}-\sum_{m=1}^{N} D_{m}^{(4)}-\sum_{m=1}^{N} D_{m}^{(6)}-\sum_{m=1}^{N} D_{m}^{(8)} \\
& -N\left(H_{\text {out }}-\frac{\stackrel{N}{N_{d}}}{\mu_{0}}\right)
\end{aligned}
$$

When $\theta=90^{\circ}$,

$$
\begin{aligned}
E\left(90^{\circ}\right) & =-\frac{J}{2}\left[N Z_{0}+2(N-1) Z_{1}\right]-\frac{\omega}{4}\left[N \Phi_{0}+2(N-1) \Phi_{1}\right] \\
& -N\left(H_{i n}-\frac{N_{d}}{\mu_{0}}\right)
\end{aligned}
$$

When $E(0)>E(90)$,

$$
\begin{aligned}
\frac{3 \omega}{4}\left[N \Phi_{0}+2(N-1) \Phi_{1}\right]+N\left(H_{\text {in }}-H_{\text {out }}\right) & >\sum_{m=1}^{N} D_{m}^{(2)}+\sum_{m=1}^{N} D_{m}^{(4)} \\
& +\sum_{m=1}^{N} D_{m}^{(6)}+\sum_{m=1}^{N} D_{m}^{(8)}
\end{aligned}
$$

When $E(0)<E(90)$,

$$
\begin{aligned}
\frac{3 \omega}{4}\left[N \Phi_{0}+2(N-1) \Phi_{1}\right]+N\left(H_{\text {in }}-H_{\text {out }}\right) & <\sum_{m=1}^{N} D_{m}^{(2)}+\sum_{m=1}^{N} D_{m}^{(4)} \\
& +\sum_{m=1}^{N} D_{m}^{(6)}+\sum_{m=1}^{N} D_{m}^{(8)}
\end{aligned}
$$

According to above equations, the in plane or perpendicular orientation is preferred depending on the marginal value of

$$
\sum_{m=1}^{N} D_{m}^{(2)}+\sum_{m=1}^{N} D_{m}^{(4)}+\sum_{m=1}^{N} D_{m}^{(6)}+\sum_{m=1}^{N} D_{m}^{(8)}
$$

given by following equation.

$$
\sum_{m=1}^{N} D_{m}^{(2)}+\sum_{m=1}^{N} D_{m}^{(4)}+\sum_{m=1}^{N} D_{m}^{(6)}+\sum_{m=1}^{N} D_{m}^{(8)}=\frac{3 \omega}{4}\left[N \Phi_{0}+2(N-1) \Phi_{1}\right]+N\left(H_{\text {in }}-H_{\text {out }}\right)
$$


For $\operatorname{sc}(001)$ with $H_{\text {in }}=H_{\text {out }}$

$$
\sum_{m=1}^{N} D_{m}^{(2)}+\sum_{m=1}^{N} D_{m}^{(4)}+\sum_{m=1}^{N} D_{m}^{(6)}+\sum_{m=1}^{N} D_{m}^{(8)}=6.284 N+0.491
$$

The anisotropy energy of a thin film is given by following equation.

$$
\begin{aligned}
E(0)-E\left(90^{\circ}\right) & =\frac{3 \omega}{4}\left[N \Phi_{0}+2(N-1) \Phi_{1}\right]-\sum_{m=1}^{N} D_{m}^{(2)}-\sum_{m=1}^{N} D_{m}^{(4)}-\sum_{m=1}^{N} D_{m}^{(6)}-\sum_{m=1}^{N} D_{m}^{(8)} \\
& +N\left(H_{\text {in }}-H_{\text {out }}\right)
\end{aligned}
$$

For $H_{\text {out }}=0$,

$$
\frac{E(0)-E(90)}{\omega}=16.284 N-64.51
$$

When $N \geq 4, \mathrm{E}(0)>\mathrm{E}\left(90^{\circ}\right)$ and perpendicular orientation is preferred. When $N \leq$ $3, \mathrm{E}(0)<\mathrm{E}\left(90^{\circ}\right)$ and in plane orientation is preferred.

For bcc $(001) \Phi_{0}=5.8675, \Phi_{1}=2.7126$

$$
\frac{E(0)-E(90)}{\omega}=18.47 N-69.07
$$

Therefore, the marginal values of number of layers for bcc(001) is same as those for $\operatorname{sc}(001)$.

Using $\mathrm{Z}_{0}=4$ and $\mathrm{Z}_{1}=1$ for $\operatorname{sc}(001)$ ferromagnetic film, $\frac{J}{\omega}=10$ and above other values in equation (1)

$$
\begin{aligned}
\frac{E(\theta)}{\omega}= & -18.95 N+10.082+(3.142 N+0.246) \cos 2 \theta \\
& -N\left[\cos ^{2} \theta\left(30+20 \cos ^{2} \theta+10 \cos ^{4} \theta+5 \cos ^{6} \theta\right)\right. \\
& +10 \sin \theta+10 \cos \theta+10 \sin 2 \theta]
\end{aligned}
$$

3-D graph of energy versus $\mathrm{N}$ and $\theta$ is shown in Figure 2.

For $N=5$

$$
\begin{aligned}
\frac{E(\theta)}{\omega}= & -84.67 N+15.96 \cos 2 \theta \\
& -5\left[\cos ^{2} \theta\left(30+20 \cos ^{2} \theta+10 \cos ^{4} \theta+5 \cos ^{6} \theta\right)\right. \\
+ & 10 \sin \theta+10 \cos \theta+10 \sin 2 \theta]
\end{aligned}
$$

Graph between energy and $\theta$ is given in Figure 3 .

For bcc(001) ferromagnetic thin films, $\mathrm{Z}_{0}=0, \mathrm{Z}_{1}=4, \Phi_{0}=5.8675, \Phi_{1}=2.7126$

$$
\begin{aligned}
\frac{E(\theta)}{\omega}= & -28.6 N+39.322+(4.235 N-2.034) \cos 2 \theta \\
& -N\left[\cos ^{2} \theta\left(30+20 \cos ^{2} \theta+10 \cos ^{4} \theta+5 \cos ^{6} \theta\right)\right. \\
& +10 \sin \theta+10 \cos \theta+10 \sin 2 \theta]
\end{aligned}
$$




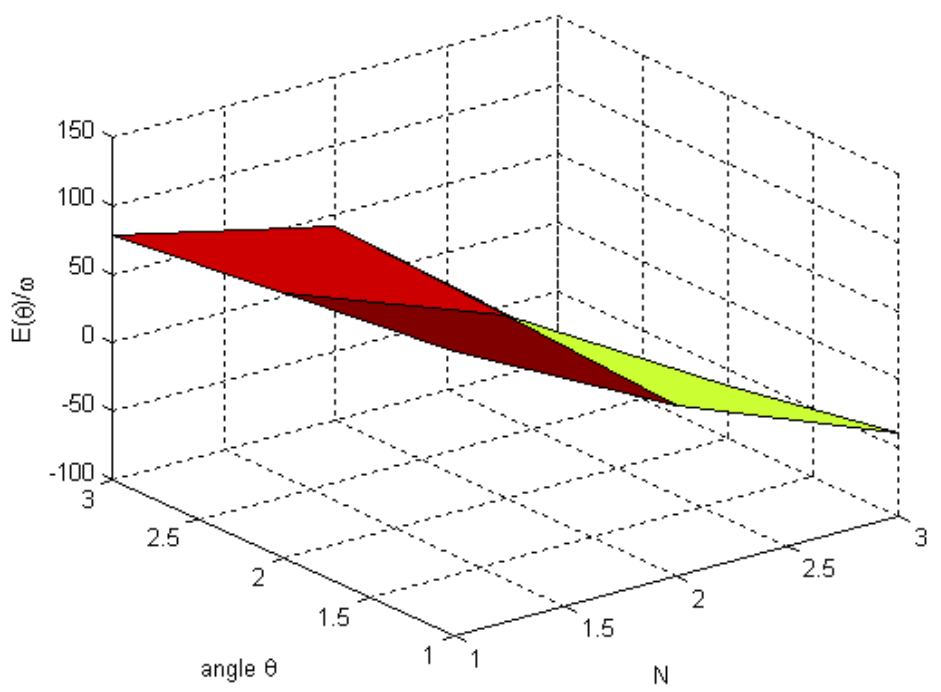

Figure 2 3-D graph of energy versus $\mathrm{N}$ and $\theta$ (radians) for sc(001) ferromagnetic film

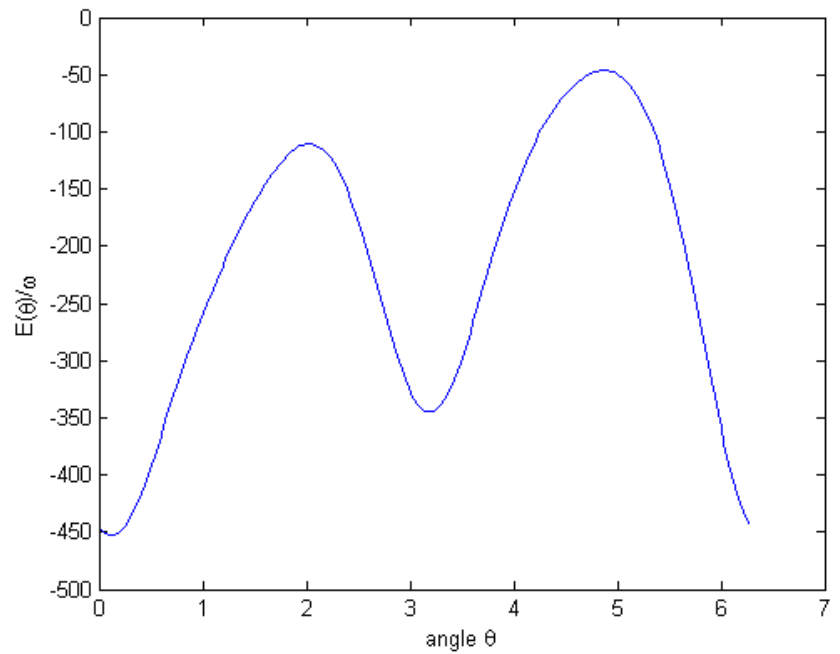

Figure 3 Graph between energy and $\theta$ (radians) for $\mathrm{N}=5$ of $\mathrm{sc}(001)$ ferromagnetic film

3 -D graph of energy versus $\mathrm{N}$ and $\theta$ is shown in Figure 4.

For $\mathrm{N}=5$,

$$
\begin{aligned}
\frac{E(\theta)}{\omega}= & -103.68+19.141 \cos 2 \theta \\
& -5\left[\cos ^{2} \theta\left(30+20 \cos ^{2} \theta+10 \cos ^{4} \theta+5 \cos ^{6} \theta\right)\right. \\
& +10 \sin \theta+10 \cos \theta+10 \sin 2 \theta]
\end{aligned}
$$




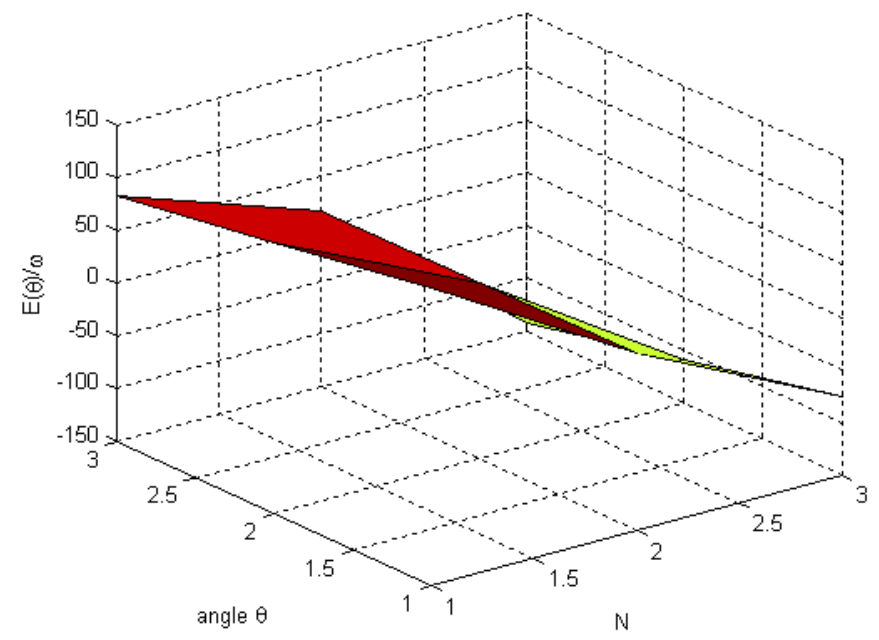

Figure $4 \quad 3-\mathrm{D}$ graph of energy versus $\mathrm{N}$ and $\theta$ (radians) for $\mathrm{bcc}(001)$ ferromagnetic film

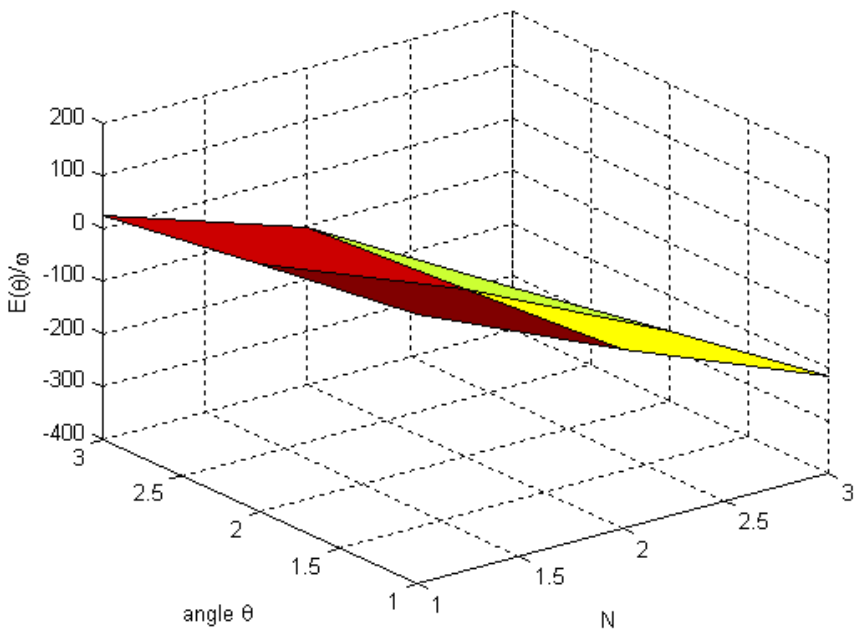

Figure 5 3-D graph of energy versus $\mathrm{N}$ and $\theta$ (radians) for $\mathrm{fcc}(001)$ ferromagnetic film.

For fcc(001) ferromagnetic thin films, $\mathrm{Z}_{0}=4, \mathrm{Z}_{1}=4, \Phi_{0}=9.0336, \Phi_{1}=1.4294$

$$
\begin{aligned}
\frac{E(\theta)}{\omega}= & -48.51 N+39.643+(4.46 N-1.072) \cos 2 \theta \\
& -N\left[\cos ^{2} \theta\left(30+20 \cos ^{2} \theta+10 \cos ^{4} \theta+5 \cos ^{6} \theta\right)\right. \\
& +10 \sin \theta+10 \cos \theta+10 \sin 2 \theta]
\end{aligned}
$$

3-D graph of energy versus $\mathrm{N}$ and $\theta$ is shown in Figure 5. 


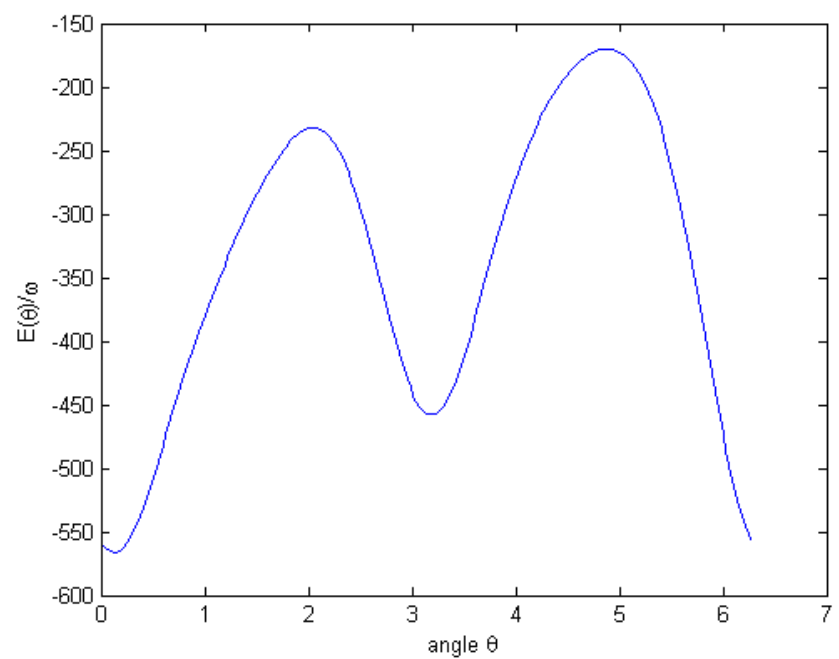

Figure 6 Graph between energy and $\theta$ (radians) for $\mathrm{N}=5$ of $\mathrm{fcc}(001)$ ferromagnetic film.

For $\mathrm{N}=5$,

$$
\begin{aligned}
\frac{E(\theta)}{\omega}= & -203+21.23 \cos 2 \theta \\
& -5\left[\cos ^{2} \theta\left(30+20 \cos ^{2} \theta+10 \cos ^{4} \theta+5 \cos ^{6} \theta\right)\right. \\
& +10 \sin \theta+10 \cos \theta+10 \sin 2 \theta]
\end{aligned}
$$

Graph between energy and $\theta$ is given in Figure 6 .

According to these graphs, first minimum value of $\frac{E(\theta)}{\omega}$ for sc(001), bcc(001) and fcc $(001)$ are $-460,-470$ and -570 , respectively. Therefore, fcc $(001)$ can be oriented easily in the easy direction compared with other two. Easy $\left(\theta=7.2^{\circ}\right)$ and hard $(\theta=$ $\left.114^{\circ}\right)$ directions for all three types with five layers remain same. But the maximum and minimum energies are different for three different types. Also the maximum and minimum energy in each graph gradually increases with angle.

\section{Conclusion}

The magnetic energy of ultra-thin films with $\mathrm{N}=1$ to 5 layers mainly depends on the azimuthual direction of magnetic moment orientation. The effect of stress induced anisotropy and the out of plane demagnetization factor on the Heisenberg Hamiltonian has been taken into account in addition to other magnetic factors. Films of $\operatorname{sc}(001)$ and bcc(001) ferromagnetic with $\mathrm{N} \leq 3$ and $\mathrm{N} \geq 4$ layers can be preferably oriented for in plane and perpendicular directions, respectively. Easy and hard directions of all $\operatorname{sc}(001), \operatorname{bcc}(001)$ and $\operatorname{fcc}(001)$ with $\mathrm{N}=5$ were found to be $\theta=7.2^{\circ}$ and $\theta=114^{\circ}$, respectively. Ultra-thin films with $\mathrm{N}=1$ or 2 can be easily oriented in $\theta=279^{\circ}$ direction. 


\section{References}

Hegde H, Samarasekara P and Cadieu F.J 1994a. Nonepitaxial sputter synthesis of aligned strontium hexaferrites, SrO.6(Fe2O3), films, J. Appl. Phys., 75(10): 6640-6642

Hegde H, Samarasekara P, Rani R, Nanavarathna A, Tracy K, and Cadieu FJ 1994b. Sputter synthesis of $\mathrm{TbCu} 7$ type $\mathrm{Sm}(\mathrm{CoFeCuZr})$ films with controlled easy axis orientation, J. Appl. Phys. 76(10): 6760-6762

Lederman David, Ricardo Ramirez and Miguel Kiwi 2004. Monte Carlo simulations of exchange bias of ferromagnetic thin films on FeF2(110), Phys. Rev. B70: 184422 $1-7$

Martin J. Klein and Robert S. Smith 1951. Thin ferromagnetic films, Phys. Rev. 81: $378-380$

Navarathna A, Samarasekara P, Hegde H, Rani R and Cadieu FJ 1994. Nitriding studies of aligned high anisotropy ThMn12-type NdFe11Co1-yMoyN film samples, J.Appl.Phys. 76(10): 6068

Samarasekara P, Rani R, Cadieu FJ and Shaheen SA 1996. Variable texture NiOFe2O3 ferrite films prepared by pulsed laser deposition, J. Appl. Phys. 79(8): 5425-5427

Samarasekara P and Cadieu FJ 2001. Magnetic and Structural Properties of RF Sputtered Polycrystalline Lithium Mixed Ferrimagnetic Films, Chinese J. Phys. 39(6): 635-640

Usadel KD and Hucht A 2002. Anisotropy of ultrathin ferromagnetic films and the spin reorientationh transition, Phys. Rev. B 66: 024419 1-6 